\title{
TRANSPORTE DE VOZ (VoIP) SOBRE REDES IPv4 e IPv6
}

\section{CARRYING VOICE ON IPv6 AND IPv4 NETWORKS}

\section{ABSTRACT}

The present paper attempts to evaluate VoIP performance by comparing its operation on both IPv6 and IPv4 networks; the architectures that support this service are also presented. In order to obtain results that truly represent those of real networks, the experiments are based not only on simulations but also on real-network implementations. One of the concluding findings suggests that VoIP6 exhibits better performance than IPv4 in terms of jitter and delay.

Keywords: VoIP, IPv6, QoS, SIP, H.323, Restricted Routing

\section{RESUMEN}

El presente artículo tiene como finalidad evaluar el comportamiento de la VoIP en redes IPv6 y compararlo con IPv4, de igual manera presentar las arquitecturas que la soportan. Con el fin de que los resultados sean los más cercanos a la realidad, el artículo no solo se soporta en simulaciones si no en implementaciones reales. Dentro de las conclusiones más sobresalientes se encontró que la VoIP6 presenta mejor rendimiento ante el Jitter y el retardo que VoIPv4.

Palabras clave: VoIP, IPv6, QoS, SIP, H.323, Enrutamiento Restringido.

\section{Fausto Alexander Gamboa}

Ingeniero de Sistemas, Egresado Universidad Distrital "Francisco José de Caldas". Estudiante Especialización en Teleinformática. Grupo investigación Internet Inteligente. fagamboaq@correo.udistrital.edu. co

\section{Octavio José Salcedo Parra}

Ingeniero de Sistemas, MSc. en Teleinformática de la Universidad Distrital, Msc. en Economía, se desempeña como docente de planta de la Universidad Distrital "Francisco José de Caldas" ojsalcedop@udistrital.edu.co

Tipo: Artículo reporte de caso

Fecha de Recepción: Agosto 30 de 2011 Fecha de Aceptación: Octubre 27 de 2011 


\section{INTRODUCCIÓN}

Los esquemas tradicionales de transmisión de voz han perdido validez, la tendencia de las redes actuales se orienta hacia la convergencia de servicios sobre una única red, esto trae implícitamente un beneficio económico sobre las empresas. La RTPC (Red Telefónica Pública Conmutada) garantiza el servicio pero se desperdicia ancho de banda. Se hace necesario migrar el servicio de voz a una tecnología que asegure la QoS gestionando de forma eficiente el espectro, además debe coexistir con otros servicios y tecnologías para garantizar convergencia, integración y unificación de servicios. La voz requiere una alta calidad de servicio, debido a que necesita intercomunicación y respuesta casi inmediata de extremo a extremo de la red sobre la cual se esté implementando. En la actualidad la VoIP se soporta sobre la versión del protocolo IPv4, no obstante debido a la inminente migración a la versión 6 , es importante que se generen estudios e investigaciones que permitan predecir su funcionamiento en este tipo de redes y las posibles ventajas y/o desventajas que puedan derivarse de la misma. El presente paper presenta los resultados encontrados cuando se implementa VoIPv6.

El artículo se divide de la siguiente manera: en la primera parte se mostrará el estado del arte, las tecnologías y protocolos empleados en este tipo de transmisiones. En la segunda parte se definirán los escenarios sobre los cuales se hacen las pruebas de funcionamiento y comportamiento, en el primer escenario se observará el desempeño del servicio de voz sobre un escenario LAN con equipos reales, en el segundo escenario se tiene una simulación de una WAN en OPNET, en la cual se compara el comportamiento de IPv4 contra IPv6 para la voz. Por último con base en los resultados obtenidos de las pruebas se analizan y presentan las conclusiones más representativas encontradas a lo largo de la investigación.

\section{ESTADO DEL ARTE}

Para transportar VoIP, la señal analógica se muestra, se cuantifica y se codifica, para convertirla en paquetes de datos formando un datagrama IP, en el nodo destino se realiza el procedimiento inverso.

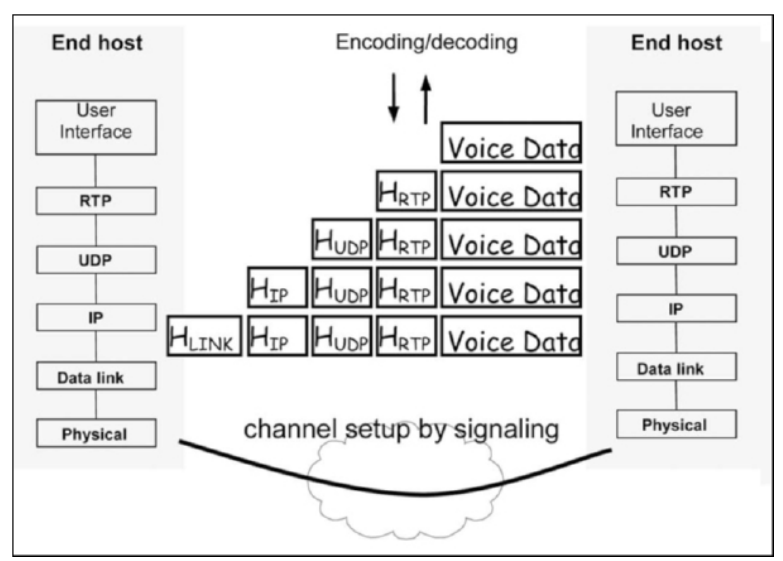

Fig. 1. Codificación de Voz Sobre IP [1]

En la VoIP, uno de los primeros protocolos en ser usados fue el estándar de la ITU H323, que cubre la mayor parte de los mecanismos necesarios para la integración de la voz (ver Fig. 1). La VoIP/H323 [2] tiene como objetivo primordial facilitar y asegurar la interoperabilidad entre equipos de diversos fabricantes, además de establecer mecanismos para los silencios, incluir técnicas de compresión, direccionamiento y establecimiento de elementos que permiten la interconectividad con la red telefónica conmutada (RTC) tradicional. La pila de protocolos de H.323 es la mostrada en la Fig. 2.

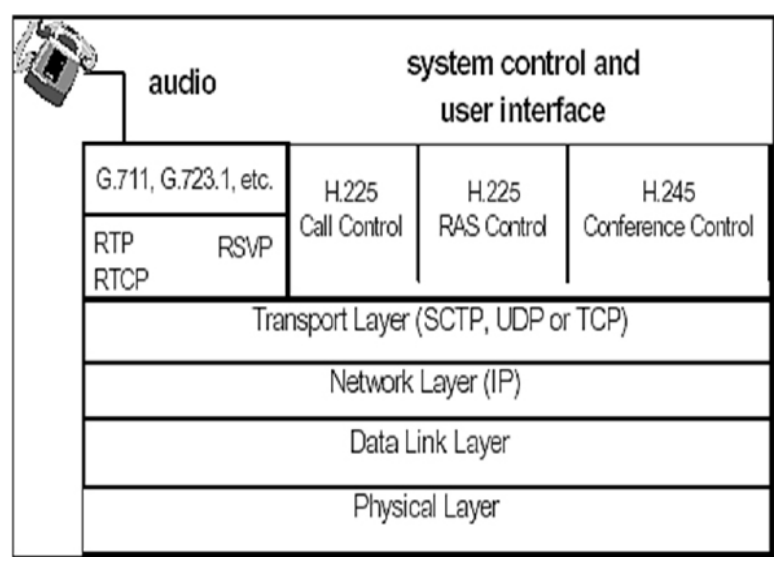

Fig. 2. Pila de protocolos H.323 [3] 
Específicamente, RTP (Protocolo de Transporte en Tiempo Real) y RTCP (Protocolo de Control en Tiempo Real) son los encargados del transporte multimedia en tiempo real y su control. RSVP (Protocolo de Reservación) segmenta los paquetes de datos grandes y se encarga de priorizar el procesamiento cuando hay una congestión en un nodo. Los códecs (G.711, G.723.1, etc.) son los estándares para la compresión de la voz. H.225 Call Control es usado para conectar dos participantes, después del establecimiento; H.225 RAS Control (registro, admisión y estado) es un protocolo de comunicaciones que permite a una estación H323 localizar a otra a través de una entidad llamada Gatekeeper. [3]

Otro estándar que garantiza el establecimiento, mantenimiento y finalización de sesiones de voz es SIP (Protocolo de Inicio de Sesiones), definido en el RFC 3261 de la IETF y es un protocolo de la capa de aplicación [4] cuya ventaja principal con respecto al H.323 es su sencillez para la realización de llamadas.

El protocolo SIP, cuya pila de componentes se puede observar en la Fig. 3, se concentra en el establecimiento, modificación y terminación de las sesiones, y se complementa entre otros con el SDP (Protocolo de descripción de sesión), que describe el contenido multimedia de la sesión (por ejemplo especifica el tipo de códec a utilizar en la comunicación). También se complementa con el RTP, donde éste es el verdadero portador para el contenido de voz y vídeo que intercambian los participantes en una sesión establecida.

SIP fue diseñado de acuerdo al modelo de Internet, por el grupo de trabajo MMUSIC (Multiparty multimedia session control) del IETF (Internet engineering task force), con el fin de estandarizar sesiones interactivas de usuario, con elementos multimedia; es un protocolo de señalización extremo a extremo que implica que toda la lógica es almacenada (incluyendo el estado de la conexión) en los dispositivos finales (salvo el enrutado de los mensajes SIP).

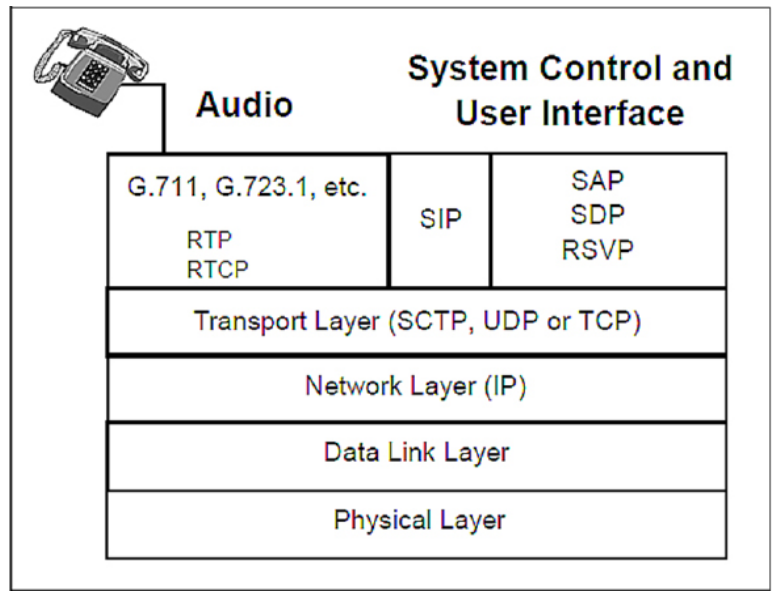

Fig. 3. Pila de Protocolo SIP [3]

La cabecera del protocolo consta de los campos mostrados en la tabla 1.

Tabla1. Cabecera protocolo SIP.

\begin{tabular}{|l|l|}
\hline Via & $\begin{array}{l}\text { Indica el transporte usado para el envío e identi- } \\
\text { fica la ruta del request, }\end{array}$ \\
\hline From & Especifica la dirección origen de la petición. \\
\hline To & $\begin{array}{l}\text { Se usa para dar la dirección del destinatario de } \\
\text { la petición. }\end{array}$ \\
\hline Call-Id & $\begin{array}{l}\text { Es un Id único para cada llamada, contiene la di- } \\
\text { rección del host. }\end{array}$ \\
\hline Cseq & $\begin{array}{l}\text { Inicia con un número aleatorio e identifica cada } \\
\text { petición de forma secuencial. }\end{array}$ \\
\hline Contact & $\begin{array}{l}\text { Contiene una (o más) dirección que se pueden } \\
\text { usar para contactar al usuario. }\end{array}$ \\
\hline User Agent & $\begin{array}{l}\text { Contiene el agente cliente que realiza la comu- } \\
\text { nicación. }\end{array}$ \\
\hline
\end{tabular}

Para que la transición hacia IPv6, mediante el protocolo SIP, sea una solución completa tiene que manejar la capa de señalización y la capa de sesión. Aunque SIP no extendido, puede manejar redes heterogéneas dentro de las diferencias fundamentales entre este y H.323 se encuentran las especificadas en la tabla 2 [5]. 
Tabla 2. Diferencias entre H.323 y SIP[6].

\begin{tabular}{|l|c|c|}
\hline \multicolumn{1}{|c|}{ Parámetro } & H-323 & SIP \\
\hline Codificación & Binaria (ASN.1) & Textual (SigComp) \\
\hline Formatos & $\begin{array}{c}\text { Serier G.XXX y } \\
\text { H.XXX, MPEG, GSM }\end{array}$ & Tipos MIME-IANA \\
\hline Ampliabilidad & Campos reservados & Métodos, cabeceras \\
\hline Autenticación & $\begin{array}{c}\text { H.235 } \\
\text { (Puede usar TLS) }\end{array}$ & Análogo a HTTP \\
\hline Localización & $\begin{array}{c}\text { Gatekeeper } \\
\text { (puede usar DNS) }\end{array}$ & \begin{tabular}{c} 
DNS \\
\hline Transporte
\end{tabular} \\
\hline
\end{tabular}

Consecuentemente:

- En H.323 se especifican servicios, mientras que SIP es un protocolo de señalización para dar base a servicios.

- H.323 enmarca un conjunto amplio de protocolos cuya implementación es obligatoria.

- En H.323 es más completa y compleja la negociación de capacidades.

- Los Mecanismos de gestión y administración de la red son definidos en H.323.

- En la arquitectura SIP son ortogonales las funciones y servicios como garantía de calidad, directorio o descripción.

- SIP está integrado en la infraestructura web y proporciona servicios de mensajería instantánea.

- Los mecanismos de detección de bucles, espirales y otros errores de configuración de la red que tiene SIP son mejores.

- El inicio de llamadas es más rápido con SIP.

\section{CALIDAD DE SERVICIO}

\subsection{RSVP}

Este protocolo se encarga del control del sistema de envío de paquetes entre el emisor y receptor (también en los nodos intermedios) su función es reservar recursos en cada nodo de la ruta. Una sesión RSVP consta de dirección de destino, un identificador del protocolo de internet IP y un puerto de destino. Cada emisor cuando utiliza RSVP intenta negociar los recursos a lo largo de toda la ruta hasta el receptor, pero sólo se le asignaran si en estos nodos intermedios tiene prioridad como solicitante. Cuando se consigue la reserva y esta es confirmada, se envían los datos. Para la negociación es necesario que haya autorización en los nodos y que estos tengan los recursos para atenderla.

RSVP puede ser utilizado tanto por hosts como por nodos con el fin de pedir o entregar niveles específicos de calidad de servicio (QoS) para los flujos de datos de las aplicaciones. RSVP define además como se deben liberar los recursos reservados una vez que estos se han usado y se ha terminado la transmisión de los datos.

Como ventaja de este mecanismo de QoS se tiene que los recursos de la ruta están estrictamente garantizados. La desventaja es que introduce una sobrecarga, que en las redes amplias, como Internet podría volverse impracticables, esto dado a que estas están compuestas por dispositivos heterogéneos en cuanto a clases y fabricantes.

\subsection{RTP}

Utilizado para la transmisión de información en tiempo real. Fue desarrollado por el grupo de trabajo de transporte de audio y video del IETF, se público como estándar por primera vez en 1996 en la RFC 1889, y actualizado posteriormente en 2003 en la RFC 3550, que constituye el estándar de Internet STD 64. Inicialmente fue publicado como un protocolo multicast, aunque su uso se propago a aplicaciones unicast. Se usa frecuentemente en sistemas de streaming, junto a RSTP, videoconferencia y sistemas push to talk (en colaboración con H.323 o SIP).

Por otro lado la RFC 3711, define SRTP (Secure Real-time Transport Protocol) como una extensión del perfil de RTP para conferencias de audio y vídeo que puede usarse opcionalmente para proporcionar confidencialidad, autenticación de mensajes y protección de reenvío para flujos de audio y vídeo. En la Fig. 4 se puede observar la estructura del encabezado RTP. 


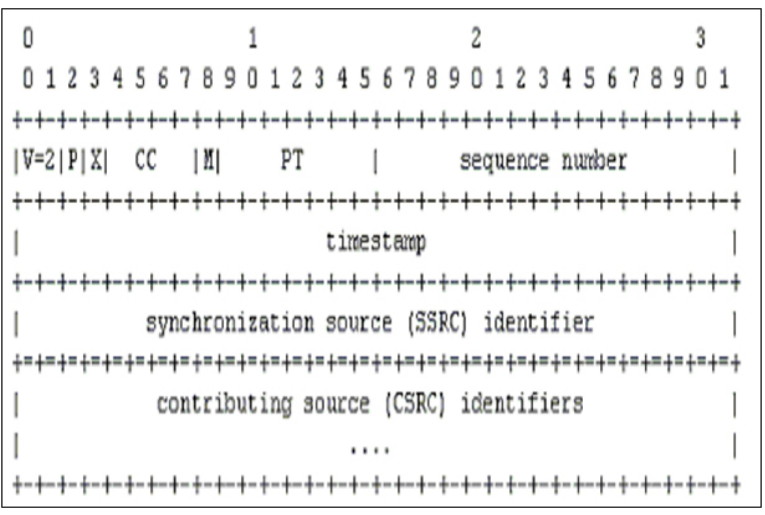

Fig. 4. Encabezado RTP. [7]

\subsection{Mecanismos de QoS}

Para asegurar la calidad en la red, se hace necesario implementar mecanismos que ayuden a lograrla, dentro del marco de posibilidades existentes, se encuentran las siguientes:

Señalización: está ligada implícitamente al estándar escogido, ya sea H.323 o SIP, pues estos dos son los encargados de realizarla.

QoS basado en routing: Al ser el transporte de voz un servicio en tiempo real, se deben implementar en la red políticas de enrutamiento, para lograrlo se debe priorizar el tráfico de voz sobre el tráfico de datos, esto se logra en IPv6 a través de los campos de clase de tráfico (8 bits) y etiqueta de flujo (20 bits.

La "clase de tráfico" está disponible para usarse por nodos origen a fin de identificar y distinguir entre las diferentes clases o prioridades de paquetes IPv6.

La "etiqueta de flujo" puede ser usado por un origen para etiquetar secuencias de datos para los cuales solicita un manejo especial por parte de los dispositivos intermedios IPv6, entre los que se encuentran la calidad de servicio no estándar o el servicio en tiempo real, muy útil para asegurar la QoS de la transmisión de voz.
Desde el punto de vista teórico se han establecido algunas ventajas de VoIPv6 sobre VoIP4 las cuales están resumidas en la tabla 3.

Tabla 3. Beneficios VoIPv6 respecto a VoIPv4

\begin{tabular}{|l|c|c|}
\hline \multicolumn{1}{|c|}{ Beneficio } & $\mathrm{IPv} 4$ & $\mathrm{IPv} 6$ \\
\hline $\begin{array}{l}\text { Integridad punto a punto de la señalización } \\
\text { de VoIP. }\end{array}$ & & $\mathrm{X}$ \\
\hline Seguridad (Escucha disimulada) & & $\mathrm{X}$ \\
\hline Adaptabilidad & $\mathrm{X}$ & $\mathrm{X}$ \\
\hline Fiabilidad & & $\mathrm{X}$ \\
\hline $\begin{array}{l}\text { Alojamiento NAT } \\
\text { (Network Address Translation ) }\end{array}$ & $\mathrm{X}$ & \\
\hline Calidad de servicio QoS & $\mathrm{X}$ & $\mathrm{X}$ \\
\hline $\begin{array}{l}\text { Soporte a tráfico multimedia en tiempo } \\
\text { real }\end{array}$ & & $\mathrm{X}$ \\
\hline Movilidad & & $\mathrm{X}$ \\
\hline Configuración dinámica & & $\mathrm{X}$ \\
\hline
\end{tabular}

Se puede concluir que IPv6 proporciona mayor facilidad para clasificar los paquetes con identificadores de tráfico. Adicionalmente, el campo etiqueta de flujo tiene la ventaja de estar localizado antes de los campos de dirección, lo que ayuda a reducir los retardos en la verificación del paquete [8].

\section{ESCENARIOS DE PRUEBAS}

Con el fin de estudiar y comparar el comportamiento de las transmisiones de voz sobre IPv4 e IPv6 se plantean dos escenarios; uno de ellos se implementa desde el punto de vista real a nivel LAN y el otro mediante la herramienta de simulación Opnet a nivel WAN .

\subsection{Escenario 1}

Servicio LAN (implementación real). Esta estructura está compuesta por un servidor Asterisk soportado en el sistema operativo CentOS, dos clientes de telefonía IP, uno de ellos Debian 6 y un Windows XP sp3, con clientes de telefonía Linphone y 3CXPhone respectivamente. La topología se muestra en la Fig. 5. 


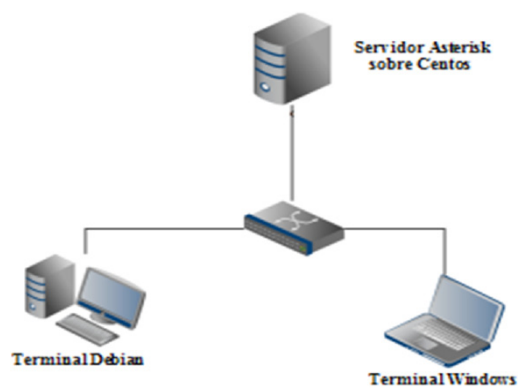

Fig. 5. Escenario de pruebas LAN.

\subsection{Escenario 2}

Simulación WAN en OPNET. En este caso se simula una red que posee dos dispositivos de capa 3, seis estaciones de trabajo y dos servidores. Se configuración 3 tipos de aplicaciones: VoIP, FTP y Video, los cuales cuentan con sus respectivos perfiles para asociarlas a las diferentes estaciones de trabajo y servidores.

Las aplicaciones FTP y video se simulan con el fin de dar carga adicional a la red, emulando un escenario real donde diferentes tipos de tráfico convergen dentro de la misma estructura. Como el análisis se centra en la aplicación de voz, se tienen cuatro estaciones de trabajo intercambiando este tipo de paquetes. El diagrama de la red se puede ver en la Fig. 6.

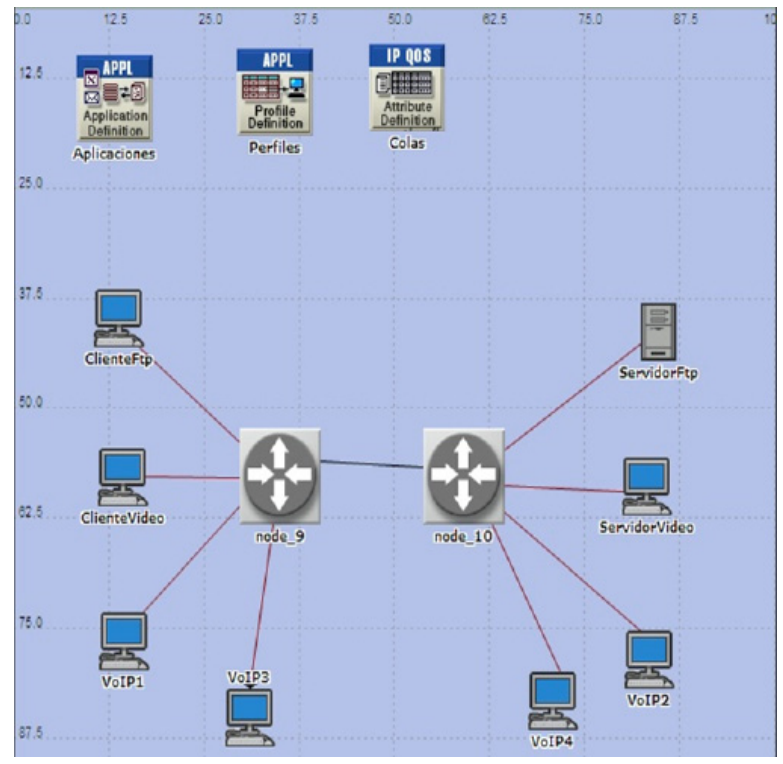

Fig. 6. Topología WAN

\section{RESULTADOS EN LA RED LAN}

Dado que las redes IP fueron concebidas para dar el "mayor esfuerzo," en IPv4 se utiliza el campo "tipo de servicio" para incluir QoS a las aplicaciones implementando Diffserv; y en IPv6 se acude a la "clase de tráfico" y "etiqueta de flujo" donde la función del primero es especificar parámetros de prioridad, retardo, rendimiento y fiabilidad, mientras que el segundo permite etiquetar todos los paquetes de la trasmisión de voz como pertenecientes al mismo flujo, permitiéndole al origen solicitar un manejo especial por parte de los enrutadores [7].

En este caso las variables analizadas fueron el retardo, jitter, Throughput.

\subsection{Retardo}

Para cuantificar esta métrica en IPv6 e IPv4, se realizaron llamadas utilizando el servicio Asteriskv6, que es un programa de software libre que implementa transmisión de voz sobre IPv6, soportado sobre SIP; de forma análoga se procedió para el caso de IPv4. Se uso el escenario 1 para la realización de esta prueba. Se tomaron como muestra diez llamadas y se realizaron diferentes capturas mediante Wireshark, donde se tomaron los paquetes recibidos y los tiempos de duración; los datos obtenidos se observan en la tabla 4.

Tabla 4. Tiempos de llamada en VoIP, v4 y v6.

\begin{tabular}{|c|c|c|}
\hline $\begin{array}{c}\text { Paquetes VoIP } \\
\text { Transmitidos } \\
\text { (Bytes) }\end{array}$ & $\begin{array}{c}\text { Tiempo de Envío } \\
\text { (en Segundos) } \\
\text { IPv4 }\end{array}$ & $\begin{array}{c}\text { Tiempo de Envío } \\
\text { (en Segundos) } \\
\text { IPv6 }\end{array}$ \\
\hline 30123 & 465,6 & 237 \\
\hline 40243 & 482,4 & 301,2 \\
\hline 50132 & 656,4 & 407,4 \\
\hline 60317 & 753 & 478,2 \\
\hline 70322 & 948 & 593,4 \\
\hline 80412 & 1084,2 & 604,8 \\
\hline 90162 & 1315,2 & 781,8 \\
\hline 100202 & 1350,6 & 883,8 \\
\hline 110371 & 1488 & 952,2 \\
\hline 120614 & 1626,6 & 1057,8 \\
\hline
\end{tabular}

Transporte de voz (VoIP) sobre redes IPv4 e IPv6 
Estos tiempos se graficaron de forma lineal para observar la mejora sustancial, que se da cuando se emplea IPv6 (Fig. 7).

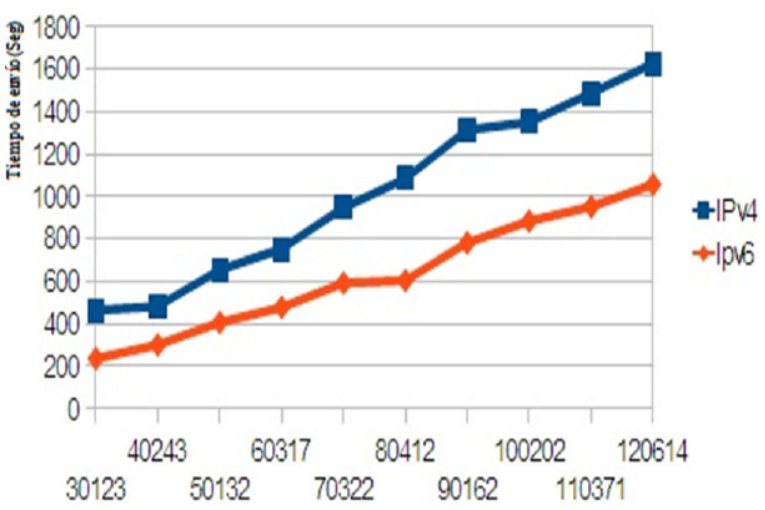

Fig. 7. Tiempos de llamada vs. Paquetes IPv4 e IPv6.

Cómo es de suponerse se observa en la gráfica anterior que a mayor número de paquetes para enviar, mayor tiempo de envío, pero se observa que la versión 6 del protocolo es más eficiente que la versión 4 en lo que se refiere al mismo número de paquetes transmitidos, pues el tiempo siempre es menor.

Para obtener el retardo de cada transferencia se utiliza la ecuación 1:

$$
\text { Re tardo }=\frac{\text { Tiempo de la llamada }}{\text { Numero de paquetes }}
$$

Los resultados se observan en la tabla 5 .

Tabla 5. Retardo en VoIP, v4 y v6.

\begin{tabular}{|c|c|c|}
\hline $\begin{array}{c}\text { Paquetes VoIP } \\
\text { Transmitidos } \\
\text { (Bytes) }\end{array}$ & $\begin{array}{c}\text { Retardo } \\
\text { (milisegundos) } \\
\text { IPv4 }\end{array}$ & $\begin{array}{c}\text { Retardo } \\
\text { (milisegundos) } \\
\text { IPv6 }\end{array}$ \\
\hline 30123 & 15,4566278 & 7,86774226 \\
\hline 40243 & 11,9871779 & 7,48453147 \\
\hline 50132 & 13,0934333 & 8,12654592 \\
\hline 60317 & 12,4840426 & 7,92811314 \\
\hline 70322 & 13,4808453 & 8,43832655 \\
\hline 80412 & 13,4830622 & 7,52126548 \\
\hline 90162 & 14,5870766 & 8,67105876 \\
\hline 100202 & 13,4787729 & 8,82018323 \\
\hline 110371 & 13,4818023 & 8,62726622 \\
\hline 120614 & 13,4859967 & 8,77012619 \\
\hline
\end{tabular}

Representando estos valores en la Fig. 8, se concluye que existe un menor retardo en la transmisión con IPv6. Esta variable oscila entre un $35 \%$ y $50 \%$ con respecto a IPv4 lo que la hace más eficiente en este tipo de redes.

En IPv4 se tienen valores entre 12 y $14 \mathrm{~ms}$, mientras que en IPv6 oscilan entre 7,4 y 8,8 ms.

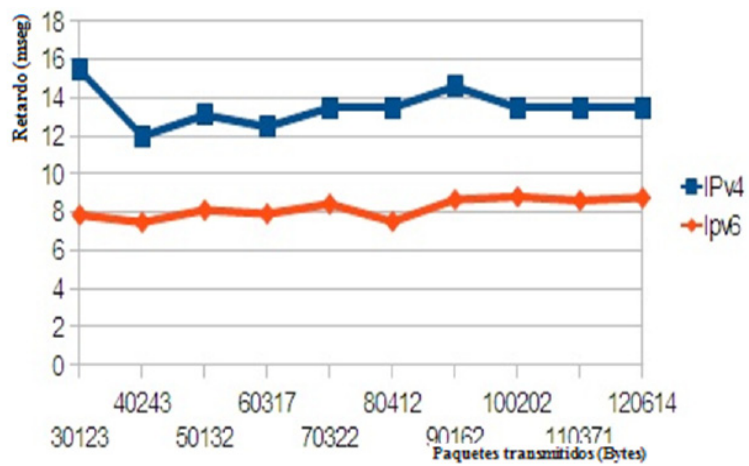

Fig. 8. Retardo IPv4 e IPv6.

\subsection{Jitter}

Para realizar la comparación del Jitter, se realizaron capturas de tráfico con Wireshark en la máquina receptora; de estas capturas se obtuvieron valores de Jitter promedio de 7,4987 ms para la versión 4 del protocolo IP y 4,983 ms para la versión 6 .

Para establecer el porcentaje de reducción, se parte del valor obtenido en la ecuación 2:

$$
\begin{aligned}
& x=\frac{4,983 m s}{7,4987 m s} * 100[\%] \\
& x=66.4515[\%]
\end{aligned}
$$

Lo que significa una reducción del Jitter en el orden de un $33.5475 \%$ al usar IPv6.

\section{RESULTADOS EN LA RED WAN}

La simulación en OPNET tiene tres escenarios distintos desde el punto de vista de las aplicaciones y protocolos, no obstante la topología física es la misma para cada caso. En el primer escenario se tiene en capa tres el protocolo IPv4 al igual que en el segundo escenario, en el tercer escenario se hace el cambio para todos los 
enlaces a IPv6; en el primer y tercer escenario se configura la calidad de servicio mínima; en el enlace entre los router se incluye un gestor de encolamiento tipo FIFO, por lo tanto no se hace priorización del distinto tráfico que llega a los nodos; en el escenario 2 se tiene encolamiento tipo PQ por el campo tipo de servicio en el encabezado IPv4, donde el mayor valor lo tiene la aplicación de voz, por encima de las aplicaciones de video y FTP.

Se ejecuta la simulación para un tiempo de $300 \mathrm{~s}$, la de voz empieza a generarse a partir del segundo 100 y es de tipo constante, adicional a esto se simula un flujo en el enlace entre los dispositivos de capa 3 que va aumentando cada 50 s. Después de realizada la simulación se comparan los resultados de los tres escenarios.

\subsection{Retardo de paquetes}

Esta variable se analiza únicamente para los paquetes de voz, en los escenarios de IPv6 e IPv4 con encolamiento FIFO; se observa que para las dos versiones del protocolo IP, una vez que empieza el tráfico, el retardo empieza a aumentar progresivamente, este retardo se analiza extremo; el resultado se puede observar en la Fig. 9.

De la simulación, se observa que en cada momento es mayor en IPv4 que en IPv6; empezando en el segundo cien, cuando inicia el tráfico de voz y aumentando fuertemente hasta estabilizarse en el segundo 300. Obteniendo valores para IPv6 entre los 10 y los $41 \mathrm{~ms}$ y para IPv4 entre 23 y $90 \mathrm{~ms}$.

\subsection{Jitter}

Se analiza para los tres escenarios, y solamente para los paquetes de voz. Los resultados obtenidos se aprecian en la Fig. 10. En el caso con priorización de tráfico, el jitter es nulo debido a que todos los paquetes de voz que llegan son procesados inmediatamente, en los otros dos escenario se ve una disminución de dicha variable hasta hacerse casi nulo, con un valor más bajo para el escenario con IPv6 que con IPv4. En la versión 6 del protocolo también se observa que converge más rápidamente a cero.

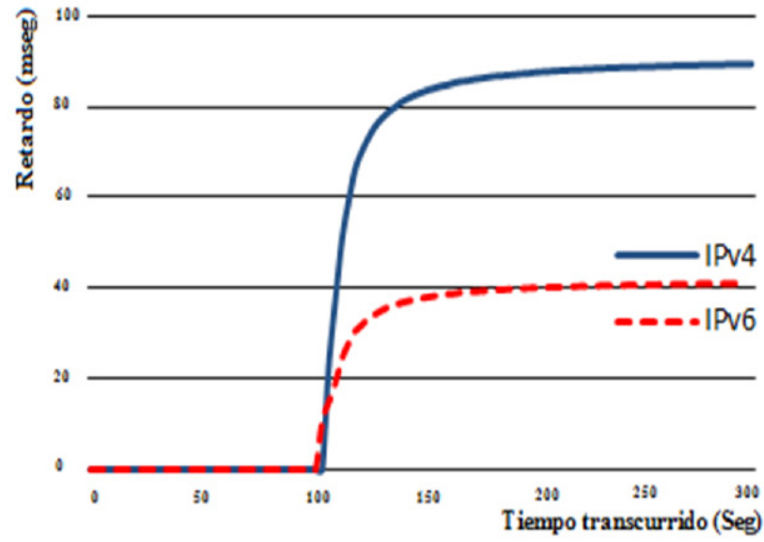

Fig. 9. Retardo de Paquetes Extremo a Extremo.

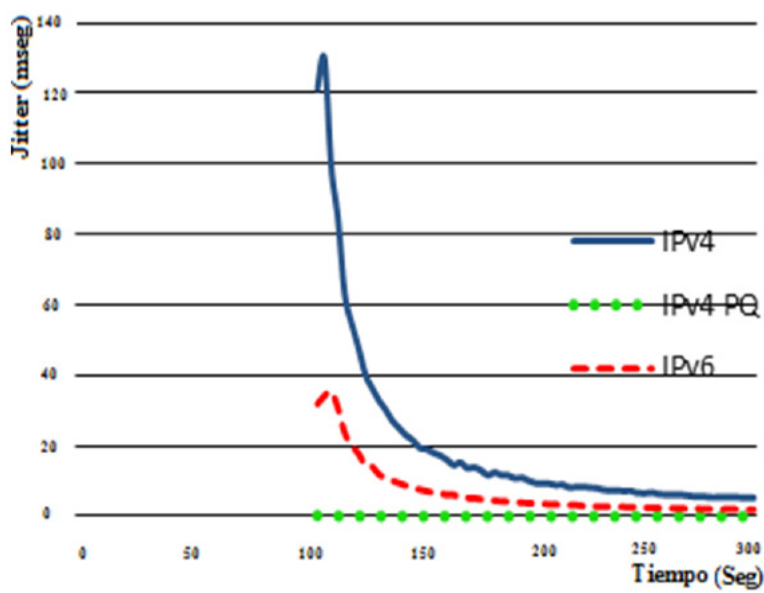

Fig. 10. Jitter.

\subsection{Throughput}

El rendimiento tomado como la medida de información se analiza específicamente para el enlace entre los dos enrutadores y se hace un estudio y comparación para los tres casos.

Se encontró que el desempeño era similar mientras no se transfiriera voz en cada escenario, una vez iniciada la transmisión de la aplicación de voz, el throughput es mayor para los escenarios con IPv4, esto dado que el encabezado de IPv4 (cuando no se utiliza el campo de "opciones") es menor que el de IPv6; mientras que el primero tiene 160 bits, el segundo tiene el doble, es decir 320, lo cual hace que los nodos tengan que procesar un número mayor de campos (ver Fig. 11). 


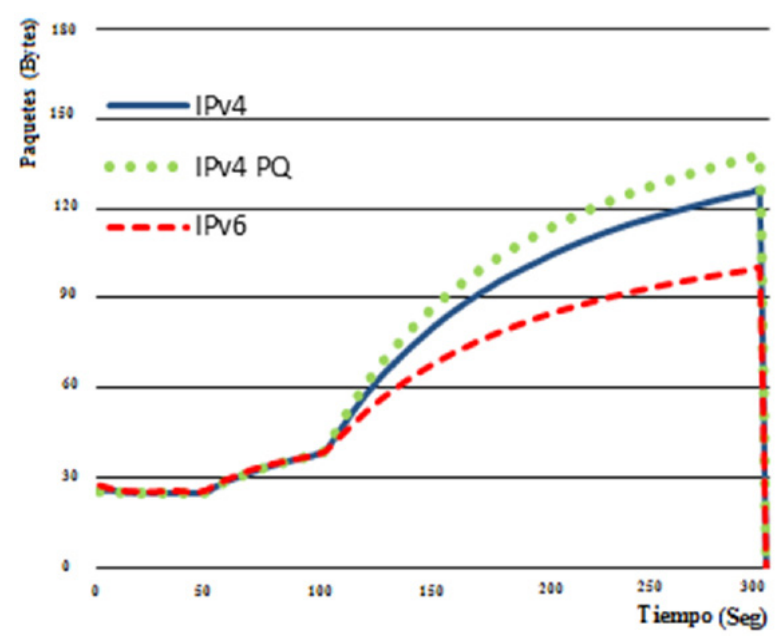

Fig. 11. Throughput Enlace Routers.

\section{CONCLUSIONES}

Al migrar de VoIP a la versión 6 del protocolo,

\section{Referencias Bibliográficas}

[1] L. Xiangyang, G. Jinhua, Security challenge and defense in VoIP infrastructures. IEEE transactions on systems, man, and cybernetics-part c: applications and reviews, vol. $37, \mathrm{~N}^{\circ}$. 6, November 2007.

[2] ITU, Recommendation H323: Visual telephone systems and equipment for local area networks provide a nonguaranteed quality of service 12/09. [En línea], consultado en diciembre 11 de 2011, disponible en: http://www.itu. int/rec/T-REC-H.323-200912-I/en.

[3] TCP/IP Tutorial and technical overview; Redbooks, IBM Chapter 20: Voice over IP. 2007.

[4] SIP: Session Initiation Protocol. RFC 3261. Junio2002. [En línea], consultado en Marzo 18 de 2011, disponible en: http://www.ietf.org/rfc/rfc3261. txt. se obtiene un mayor rendimiento en la aplicación, pues se tiene un menor Jitter y un menor retardo, lo que incrementa la experiencia de uso positiva por parte del usuario, adicional a esto se presenta una menor perdida de paquetes en general, para todo el tráfico IP.

En términos generales, sin aplicar ninguna técnica de calidad de servicio, el desempeño de IPv6 es superior a IPv4; cuando se introduce calidad de servicio a IPv4, los resultados son similares a los obtenidos en IPv6 sin ningún tipo de QoS.

La utilización de IPv6 tiene costos a nivel de procesamiento, esto debido al tamaño de la cabecera, pues los equipos tienen que procesar un número mayor de datos cuando existen cabeceras de extensión.

[5] IPv6 transition in the session initiation protocol (SIP). RFC 3264. Abril 2011. [En línea], consultado en Agosto 17 de 2011, disponible en: http://www.ietf. org/rfc/rfc3264.txt.

[6] Federico M; SIP: Session initiation protocol IRIS-MMEDIA. XV grupos de trabajo de RedIRIS, 2003.

[7] RTP profile for audio and video conferences with minimal control RFC 3551. Julio 2003. [En línea], consultado en Mayo 8 de 2011, disponible en: http://www.ietf.org/rfe/rfc3551.txt.

[8] Using the flow label field in IPv6. RFC 1809. Junio 1995, [en línea] consultado en enero de 2012, disponible: http:// www.ietf.org/rfc/rfc3264.txt 